\title{
Wall-Induced Orientational Order of a Liquid Crystal in the Isotropic Phase-an Evanescent-Wave-Ellipsometry Study
}

\author{
H. Hsiung, Th. Rasing, and Y. R. Shen \\ Department of Physics, University of California, Berkeley, California 94720 \\ (Received 6 August 1986)
}

\begin{abstract}
Using an evanescent-wave ellipsometric technique, we have studied the wall-induced orientational order in the isotropic phase of $4^{\prime}-n$-pentyl-4-cyanobiphenyl (5CB) at a silane-treated glass surface. Near the isotropic-nematic transition temperature $T_{\mathrm{NI}}$, a weak nematic layer appears at the interface with its thickness exhibiting a critical divergence as $T \rightarrow T_{\mathrm{NI}}$ according to $\left(T-T_{\mathrm{NI}}\right)^{-0.5}$. This observed pretransitional behavior is qualitatively at variance with the existing theories.
\end{abstract}

PACS numbers: $61.30 . \mathrm{Gd}, 64.70 . \mathrm{Md}, 68.45 . \mathrm{Gd}$

A properly treated solid surface can preferentially orient liquid crystal (LC) molecules in their mesophases. ${ }^{1}$ The effect is the result of anisotropic interactions between the molecules and the solid surface and is essential for the construction of many LC devices. These molecule-substrate interactions could induce an orientational order of the LC molecules near the solid surface even when the bulk LC is in the isotropic phase. Such a wall-induced pretransitional behavior has been studied theoretically, first with the Landau-de Gennes formalism $^{2-4}$ and, more recently, in terms of wetting phenome$\mathrm{na}^{5-7}$ with the wall-induced nematic order viewed as "wetting" of the isotropic LC-solid interface by a nematic layer.

Despite its theoretical and practical interest, experimental studies of the wall-induced orientational ordering have been scarce. So far, the only quantitative work reported in the literature was by Miyano ${ }^{8}$ and Tarczon and Miyano ${ }^{8}$ and van Sprang; ${ }^{9,10}$ both have used transmission ellipsometric techniques. The overall induced birefringence of the interface layer was measured as a function of temperature. Their results were analyzed and interpreted in terms of the Landau-de Gennes theory. There are obvious limitations in this approach: (1) The probe beam passes through both the interfacial and the bulk regions; any spurious birefringence induced in the bulk would be difficult to eliminate. (2) Since only the overall birefringence is measured, the local induced birefringence at the wall and the characteristic thickness of the interfacial layer cannot be deduced separately.

Aiming at eliminating these limitations so that more critical comparison with theories could be made, we have developed an improved ellipsometric technique using an evanescent probe beam in a total-reflection geometry. Consider a laser beam incident upon a LC-substrate interface from the substrate side. Total reflection occurs when the angle of incidence is above a critical angle, and the optical field on the LC side becomes evanescent. Since an evanescent optical wave is bounded at the interface, it is particularly sensitive to optical properties of the interfacial region. Furthermore, its penetration depth can be adjusted by variation of the incident angle. Thus, information about the interfacial layer at various depths can be deduced from a scan of the incident angle. This then allows a separate determination of the wallinduced order parameter and the interfacial layer thickness. In situ monitoring of the bulk LC is also made possible through a measurement of the critical angle, which depends only on the refractive index of the bulk LC relative to that of the substrate. In this respect, bulk and interfacial contributions to the properties of the reflected beam are easily distinguishable. This is particularly important for studying interfacial phenomena near a bulk transition, and, in addition, makes the study of the nematic LC-solid interface possible.

Using the evanescent-wave ellipsometry technique, we have studied the wall-induced orientational order in the isotropic phase of $4^{\prime}-n$-pentyl-4-cyanobiphenyl (5CB) at a glass surface coated with $\mathbf{N}, \mathbf{N}$-dimethyl-N-octadecyl3-aminopropyl-trimethoxysilyl chloride (DMOAP). Our technique had a sensitivity better than the transmission ellipsometric techniques by a factor of 5 or more. The induced order parameter at the wall and the interfacial layer thickness (coherence length $\xi$ ) could be determined for each temperature $T$ independently. For $T \gtrsim T_{\mathrm{NI}}$ (isotropic-nematic transition temperature), we found a weak wall-induced nematic layer at the interface; its order parameter at the wall was nearly independent of temperature, but the coherence length $\xi$ diverged according to $\left(T-T_{\mathrm{NI}}\right)^{-0.5}$ as $T \rightarrow T_{\mathrm{NI}}$. Although the observed exponent $(0.5)$ of the coherence length agrees with the value given by the Landau-de Gennes theory, the observed "complete wetting" behavior (i.e., $\xi \rightarrow \infty$ as $T \rightarrow T_{\text {NI }}$ ) with a relatively small induced order parameter is not predicted by it, nor by the wetting theories. ${ }^{5,6}$

In our experiment, the liquid crystal 5CB was obtained from BDH, Inc., and used without further purification. Its isotropic-nematic transition occurred at $35.0^{\circ} \mathrm{C}$, with a coexistence width of $\sim 100 \mathrm{mK}$. As shown in Fig. 1, the sample assembly consisted of a glass prism (Schott glass LaSF 5), a glass plate, and a 130$\mu \mathrm{m}$ layer of $5 \mathrm{CB}$ sandwiched between the prism and the 


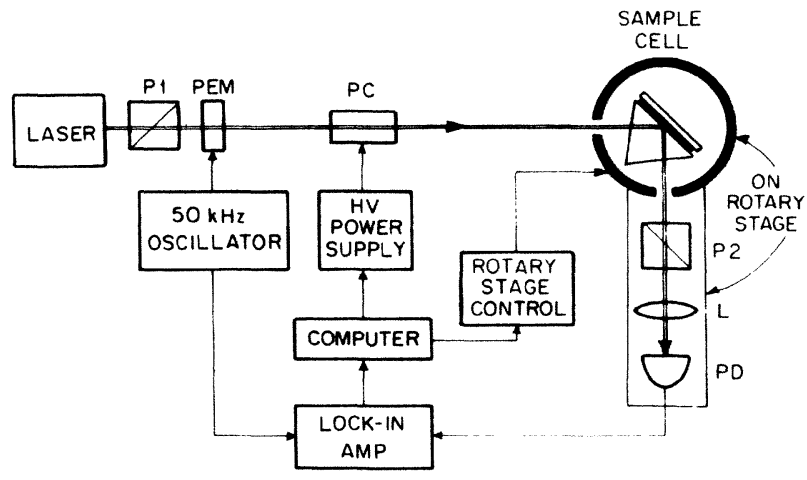

FIG. 1. Experimental arrangement. P1, polarizer; P2, analyzer; PEM, photoelastic modulator; PC, Pockels cell; L, lens; $P D$, photodiode.

plate. Both glass surfaces sandwiching the LC were coated with a monolayer of silane surfactant molecules, DMOAP, to assure homeotropic alignment for $5 \mathrm{CB}$ in the nematic phase. ${ }^{11}$ The sample assembly was situated in a two-stage oven with its temperature stabilized to $\sim 1 \mathrm{mK}$. The experimental arrangement is depicted in Fig. 1. A weak He-Ne laser beam at $\lambda=6328 \AA$ was used as the probe light. The phase difference $\Delta \phi$ between the $p$ - and $s$-polarization components of the reflected beam was measured with basically the standard high-resolution ellipsometry technique. ${ }^{12}$ We were able to achieve an accuracy of $1 \times 10^{-4} \mathrm{rad}$ in the measurements of $\Delta \phi$.

The sample cell was mounted on a rotary stage driven by a stepping motor. The beam angle of incidence $\theta$ could then be varied by a rotation of the sample cell. Angular scans of phase retardation, $\Delta \phi(\theta)$, were made for a series of temperatures at $T>T_{\mathrm{NI}}$, decreasing from high to low. Before a run at a certain $T$, ample time $\left(\sim \frac{1}{2} h\right)$ was allowed for the LC to reach thermal equilibrium. The results are shown in Fig. 2(a). The glass prism contributed a small but nonnegligible background in $\Delta \phi(\theta)$ due to its residual stress birefringence. Through measurement of the $\Delta \phi(\theta)$ of the sample cell with the LC replaced by an isotropic liquid (DowCorning 702 silicone fluid was used), the background $\Delta \phi(\theta)(\sim 0.01 \mathrm{rad})$ was determined. Over the temperature range under study, this background was roughly independent of temperature. The bulk phase transition and the isotropic-nematic coexistence region were easily recognizable through a significant change in the shape of the $\Delta \phi(\theta)$ curve and the position of $\theta_{c}$. At a given temperature $T>T_{\mathrm{NI}}, \Delta \phi\left(\theta_{c}\right)$ [see Fig. $2(\mathrm{~b})$ ] is a visual indication of the interfacial ordering effect. This quantity would vanish in the absence of an interfacial layer.

Figure 2(a) shows a monotonic increment of $\theta_{c}$ with decreasing $T$. This is due to the temperature dependence of the bulk optical dielectric constant $\varepsilon_{l}$ for the isotropic phase. The curves also exhibit clearly a $\Delta \phi\left(\theta_{c}\right)$ that
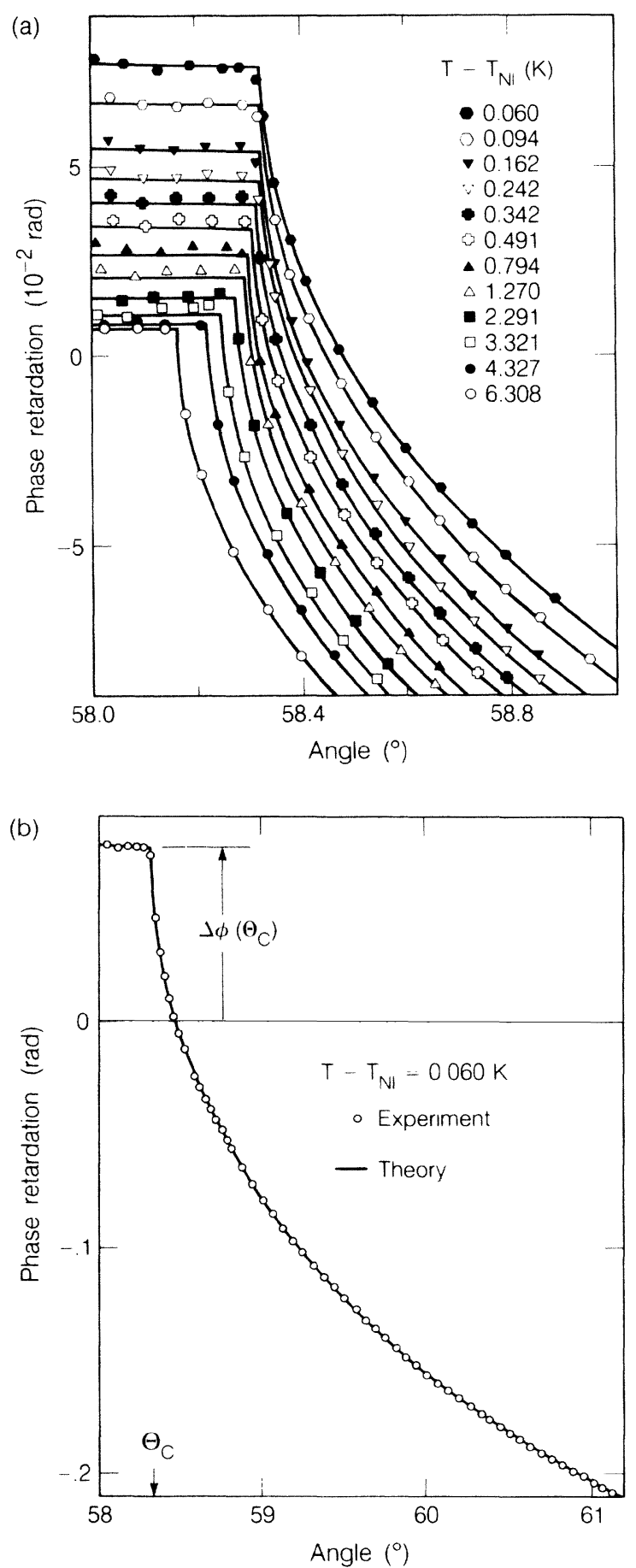

FIG. 2. (a) $\Delta \phi(\theta)$ curves at $T>T_{\mathrm{NI}}$. Only the portion around $\theta_{c}$ is displayed. Solid curves were obtained from theoretical calculations. (b) A typical $\Delta \phi(\theta)$ curve with the background subtracted (see text).

diverges as $T$ approaches $T_{\mathrm{NI}}$. The existence of a wallinduced ordering effect with a strong pretransitional behavior is thus apparent even in the raw data. The divergence of $\Delta \phi\left(\theta_{c}\right)$ as $T \rightarrow T_{\mathrm{NI}}$ is a result of the diver- 
gence of the coherence length $\xi$, as we shall discuss below.

To fit the data of $\Delta \phi(\theta)$ at a given $T$, we assumed an anisotropy in the optical dielectric constant as

$$
\begin{aligned}
\Delta \varepsilon(z) & =\varepsilon_{\|}(z)-\varepsilon_{\perp}(z) \\
& =\Delta \varepsilon_{0} \exp (-z / \xi) \quad \text { for } z \geq 0,
\end{aligned}
$$

where $z=0$ refers to the boundary surface between the LC and the glass prism, and both $\Delta \varepsilon_{0}$ and $\xi$ could be functions of temperature. This assumption can be justified from the Landau-de Gennes theory if $\Delta \varepsilon_{0}$ is sufficiently small, ${ }^{4}$ which is true in our case. We used the $4 \times 4$ matrix technique developed by Berreman and Scheffer ${ }^{13}$ to calculate the linear wave propagation in the LC medium and consequently $\Delta \phi(\theta)$, treating $\Delta \varepsilon_{0}$ and $\xi$ as adjustable parameters, and then a nonlinear leastsquares fit procedure to deduce $\Delta \varepsilon_{0}$ and $\xi$. The theoretical curves in Fig. 2(a) thus obtained are in excellent agreement with the experimental ones, with an average error estimated to be $1 \times 10^{-4} \mathrm{rad}$. An example showing the detailed fit is presented in Fig. 2(b). The critical angle for total reflection $\theta_{c}$ is determined solely by the bulk optical constants $\varepsilon_{l}$ for the LC and $\varepsilon_{g}$ for the glass through the relation $\theta_{c}=\sin ^{-1}\left(\varepsilon_{l} / \varepsilon_{g}\right)^{1 / 2}$, as long as the penetration depth $\left(>\lambda / 2 \pi \sqrt{\varepsilon_{l}}\right)$ of the optical wave is larger than the thickness $(<400 \AA$ in our case) of the interfacial layer.

We found that $\Delta \varepsilon_{0}=0.081 \pm 0.018$, which is essentially a constant in the temperature range studied. This corresponds to a temperature-independent boundary order parameter $Q_{0} \equiv \Delta \varepsilon_{0} / \Delta \varepsilon_{m}=0.077 \pm 0.017$, where $\Delta \varepsilon_{m}$ is the maximum value of $\Delta \varepsilon$ when all molecules are perfectly aligned. ${ }^{14}$ We notice that $Q_{0}$ is much smaller than the bulk order parameter $Q \geq Q_{c}\left(T-T_{\mathrm{NI}}\right) \simeq 0.33$ in the nematic phase. ${ }^{15}$ The accuracy of $\Delta \varepsilon_{0}$ was limited by the resolution of $\Delta \phi$ in our measurements. The values of $\xi$ deduced for various $T$ can be described by the function

$$
\xi(T)=6.6\left(T / T_{c}-1\right)^{-0.51 \AA,}
$$

with a standard deviation of $2.8 \AA$ (Fig. 3). The op-

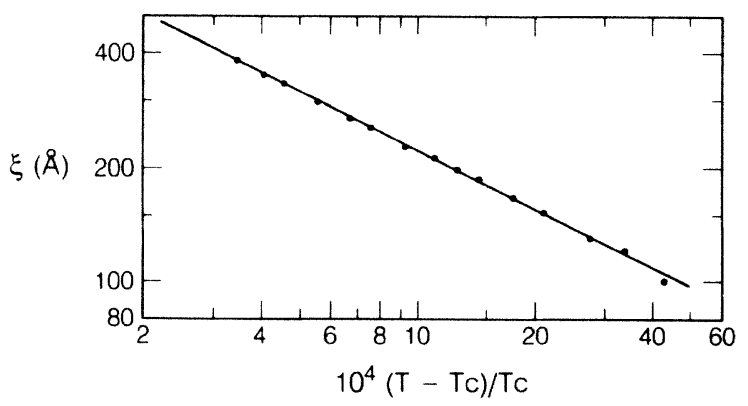

FIG. 3. Coherence length of the interfacial layer as a function of the reduced temperature (on $\log -\log$ scales). The solid line is described by Eq. (2). timum fit was obtained with $T_{c}=T_{\mathrm{NI}}-40 \mathrm{mK}$, which falls within the isotropic-nematic coexistence region $\left(T_{\text {NI }} \pm 50 \mathrm{mK}\right)$.

According to the Landau-de Gennes theory, in the limit of sufficiently weak boundary order, the coherence length should have the form $\xi=\xi_{c}=\xi_{0}\left(T / T^{\prime}-1\right)^{-1 / 2}$, where $T^{*}$ describes the bulk supercooling limit. ${ }^{4}$ For 5CB, $T^{*}=T_{\mathrm{NI}}-1.1 \mathrm{~K},{ }^{16} \xi_{0}=5-6 \AA .{ }^{9,17}$ In Eq. (2), both the exponent $\left(\simeq \frac{1}{2}\right)$ and the length scale $(6.6 \AA)$ agree closely with those of $\xi_{c}$, but the critical temperature $T_{c}$ appears to be near $T_{\mathrm{NI}}$, instead of $T^{*}{ }^{18}$ Physically, we do not expect the critical behaviors of the interfacial coherence length $\xi$ and the bulk correlation length $\xi_{c}$ to be the same. We note that $\xi_{c}$ is the average size of the nematic domains in the isotropic background, and these domains are uncorrelated. At the interface, however, an in-plane, long-range orientational correlation is established by the wall-anchoring force. Such an in-plane correlation is likely to enhance the correlation along the surface normal, and lead to a critical behavior of $\xi$ different from that of $\xi_{c}$.

Our observation that $\xi$ diverges at $T_{c}=T_{\text {NI }}$ corresponds to the complete wetting of the isotropic LC-solid interface as $T \rightarrow T_{\mathrm{NI}}$. This is not predicted by the existing theories. Both the Landau-de Gennes theory ${ }^{2-4}$ and the wetting theories ${ }^{5,6}$ have indicated that complete wetting may occur when $Q_{0} \gtrsim Q_{c}$ (bulk nematic order) as a result of a divergent growth of a plateau in the profile of $Q(z)$. We found, however, complete wetting with a relatively weak wall-induced nematic ordering $\left(Q_{0} \ll Q_{c}\right)$ without incurring any prewetting transition, at variance with theoretical prediction.

Another descrepancy between theory and experiment is in the temperature dependence of $Q_{0}$. The theories predict that $Q_{0}$ becomes weakly dependent on temperature only when the wall-anchoring force is very strong, leading to a large value of $Q_{0}\left(>Q_{c}\right)$. Experimentally, we found an approximately temperature-independent $Q_{0}$ with $Q_{0} \ll Q_{c}$. Recently, Guyot-Sionnest, Hsiung, and Shen ${ }^{19}$ have also observed a dipole layer of $8 C B$ at an 8CB-DMOAP-coated-glass interface, which does not change its character over a wide range of temperature.

Finally, we compare our results with those obtained by Miyano $^{8}$ from a similar 5CB-DMOAP-coated-glass interface. Using the Landau-de Gennes theory, he deduced a value $Q_{0}=0.169$ (with $\xi_{0}=6 \AA$ ) for the interfacial layer, which only partially wetted the isotropic LCsolid interface at $T=T_{\text {NI }}$ (i.e., $\xi$ remained finite at $T_{\mathrm{NI}}$ ), in contrast to our observation. Aside from the limitations in Miyano's measurement discussed earlier, we may attribute the differences to his use of a continuous temperature scan in the measurements and a background level as an adjustable parameter in fitting the data.

Modifications of the existing theories are clearly needed to explain our observation. Further experimental 
studies would also help to obtain a more complete picture of the wetting behavior. The wall potential can be varied systematically by different surface treatment. Liquid crystals in a homologous series bounded to the same substrate can be studied. Our technique could also allow the investigation of the LC-solid interface with the bulk LC in the nematic phase and will provide important information on how the surface ordering $Q_{0}$ varies across $T_{\text {NI. }}$. One interesting possibility is the observation of "drying" of a solid surface by an interfacial layer with $Q_{0}<Q$ (bulk). The results of the recent experiment by Faetti et al. ${ }^{20}$ indicated that this could be the case for $5 \mathrm{CB}$ on a glass substrate coated with obliquely evaporated $\mathrm{SiO}$.

This work was supported by National Science Foundation-Solid State Chemistry-Grant No. DMR8414053. We are grateful to E. I. du Pont de Nemours \& Co. for providing financial support for a research associateship for one of us (H.H.); one of us (Th. R.) acknowledges an IBM postdoctoral fellowship while participating in this project.

${ }^{1}$ See for example, J. Cognard, Alignment of Nematic Liquid Crystals and Their Mixtures (Gordon and Breach, London, 1982).

2P. Sheng, Phys. Rev. Lett. 37, 1059 (1976), and Phys. Rev. A 26, 1610 (1982).

${ }^{3}$ D. W. Allender, G. L. Henderson, and D. L. Johnson, Phys. Rev. A 24, 1086 (1981).

${ }^{4}$ A. Mauger, G. Zribi, D. L. Mills, and J. Toner, Phys. Rev. Lett. 53, 2485 (1984), and 54, 2552 (1985).

${ }^{5}$ M. M. Telo da Gama, Mol. Phys. 52, 611 (1984).

${ }^{6}$ A. Poniewierski and T. J. Sluckin, Mol. Cryst. Liq. Cryst. 111, 373 (1984); T. J. Sluckin and A. Poniewierski, Phys. Rev.
Lett. 55, 2907 (1985).

${ }^{7}$ The Landau-de Gennes theory can be transformed into the formalism of a standard wetting-transition theory. See M. Schick, Phys. Rev. Lett. 54, 2169 (1985). For a general review of wetting, see P. G. de Gennes, Rev. Mod. Phys. 57, 827 (1985).

${ }^{8}$ K. Miyano, Phys. Rev. Lett. 43, 51 (1979), and J. Chem. Phys. 71, 4108 (1979); J. C. Tarczon and K. Miyano, J. Chem. Phys. 73, 1994 (1980).

${ }^{9}$ H. A. van Sprang, J. Phys. (Paris) 44, 421 (1983), and Mol. Cryst. Liq. Cryst. 97, 255 (1983).

${ }^{10}$ Semiquantitative results on the wall-induced ordering in LC have also been obtained by $\mathrm{H}$. Mada and S. Kobayashi [Appl. Phys. Lett. 35, 4 (1979), and Mol. Cryst. Liq. Cryst. 66, $57(1981)]$

${ }^{11}$ F. J. Kahn, Appl. Phys. Lett. 22, 386 (1973).

${ }^{12}$ See for example, R. M. A. Azzam and N. M. Bashara, Ellipsometry and Polarized Light (North-Holland, Amsterdam, 1977), Ch. 5.

${ }^{13}$ D. W. Berreman and T. J. Scheffer, Phys. Rev. Lett. 25 577 (1970), and Mol. Cryst. Liq. Cryst. 11, 395 (1970).

${ }^{14}$ R. G. Horn, J. Phys. (Paris) 39, 105 (1978), and references therein.

${ }^{15}$ Attempts to fit the data by imposing a $Q_{0}$ larger than $Q_{c}$ with an exponential or any nonexponential profile $Q(z)$ have resulted in much larger errors $\left(>5 \times 10^{-4} \mathrm{rad}\right)$.

${ }^{16}$ H. J. Coles, Mol. Cryst. Liq. Cryst. 49, 67 (1978).

${ }^{17}$ T. W. Stinson and J. D. Litster, Phys. Rev. Lett. 30, 688 (1973).

${ }^{18}$ Pretransitional phenomena that show deviation from the prediction of the Landau-de Gennes theory in the vicinity of $T_{\text {NI }}$ have been reported in the literature; interpretations invoking renormalization of $T^{*}$ (towards $T_{\mathrm{NI}}$ have been proposed. See for example, E. F. Gransbergen, L. Longa, and W. H. De Jeu, Phys. Rep. 135, 195 (1986), and references therein.

${ }^{19}$ P. Guyot-Sionnest, H. Hsiung, and Y. R. Shen (Phys. Rev. Lett. 57, 2963 (1986).

${ }^{20}$ S. Faetti, M. Gatti, V. Palleschi, and T. J. Sluckin, Phys. Rev. Lett. 55, 1681 (1985). 